\title{
Agricultores familiares do Sul da Amazônia: Desafios e estratégias para inovação agroecológica de sistemas de produção
}

\section{Smallholders of South of Amazon: Challenges and strategies for innovation of agroecological their production systems}

\author{
Lucimar Santiago de Abreul, Maria Aico Watanabe ${ }^{2}$
}

\begin{abstract}
Resumo: A importância agroecológica e ambiental da Amazônia para o equilíbrio do clima e biodiversidade é mundialmente reconhecida. Porém, pouco se discute os usos dos recursos naturais pela população regional ou a conservação ambiental, a agro biodiversidade e a segurança alimentar. Entre o final de 2007 e 2016 foram conduzidas pesquisas sobre a produção agroflorestal de produtores ecológicos de Ouro Preto do Oeste, Rondônia, sul da Amazônia. Inicialmente foram entrevistados 29 agricultores de base ecológica; depois, com visitas e contatos com produtores e agentes locais, acompanhou-se a experiência e atualizaram-se os dados da pesquisa. Assim objetivou-se com este trabalho avaliar as potencialidades da diversificação agroecológica das atividades pela comparação do que cultivavam e criavam e o que poderiam cultivar e criar conforme as espécies existentes e as espécies já pesquisadas para região, analisando os desafios para a produção oriunda dessas atividades. A comparação mostra que apesar do avanço e consolidação em termos de desenvolvimento de sistemas agroflorestais, há ainda amplo espaço para a inovação e a diversificação agroecológica das atividades pelos agricultores. Atualmente há múltiplas entidades públicas e privadas na região que oferecem assistência técnica. Contudo, nem todos os agricultores tem acesso a esses serviços e a qualidade técnica exigida para a condução de sistemas agroflorestais precisam ser adequadas aos princípios da agroecologia demanda crucial dos agricultores familiares, tratando-se de requisito para o desenvolvimento sustentável local.
\end{abstract}

Palavras-chave: Agroecologia; Agricultura familiar; Ouro Preto do Oeste; Inovação tecnológica; Sistemas agroflorestais

\begin{abstract}
The agroecological and environmental importance of the Amazon for climate equilibrium and biodiversity are worldwide recognized. But little is discussed the natural resources use by regional population or the environmental conservation, agrobiodiversity and food security. Between the end of 2007 and 2016 it was conducted researches about the agroforest production of Associação de Produtores Alternatives (APA), Ouro Preto do Oeste, Rondônia, South of Amazon. Firstly it was interviewed 29 ecological-based farmers, with visits and contacts with producers and local agents followed the experience and up-dated the research data. Thus, it was objectified with this work to evaluate the potentialities of activities diversification by comparison of what cultivated and raised, what could cultivate and raise according to the existent species, and the species already researched for the region, and to evaluate the challenges for production originated from these activities. The comparison shows that in spite of agroforest systems development, there is wide space for innovation and diversification of the activities by the farmers. Currently multiple public and private entities in the region offer technical assistance. However not all the farmers have access to these services and the technical quality required for the conduction of agroforest systems need to be adequate to farmers' demand, being requirement for local sustainable development.
\end{abstract}

Key words: Agroecology; Small farming; Ouro Preto do Oeste; Technological innovation; Agroforest systems

\footnotetext{
*Autor para correspondência

Recebido para publicação em 14/04/2016; aprovado em 21/12/2016

${ }^{1}$ Doutora em Sociologia Rural, Pesquisadora da Embrapa Meio Ambiente. Jaguariúna (SP).019 33112648. lucimar.abreu@embrapa.br

${ }^{2}$ Doutora em Agronomia, Pesquisadora da Embrapa Meio Ambiente, Jaguariúna (SP). 019 33112676. aico.watanabe@embrapa.br
} 


\section{INTRODUÇÃO}

A agricultura convencional é baseada em sistemas agrícolas artificiais que se caracteriza pelo nível elevado de mecanização, pela padronização das práticas agrícolas, uso massivo de agroquímicos, dependência de insumos externos (fertilizantes químicos, agrotóxicos e combustíveis fósseis), pela incapacidade de funcionar sem grandes quantidades de água e energia, além disto promove a simplificação dos sistemas de produção. Essa agricultura implica também na redução de mão de obra, o que restringe diretamente o vínculo do ser humano com os agroecossistemas e agrava a situação de desemprego já existente nas grandes metrópoles. No entanto, experiências agroecológicas emergem em diversas localidades da Amazônia brasileira, tendo como base de suporte o desenvolvimento de sistemas agroflorestais, doravante denominado SAFs. As experiências agroecológicas são articuladas em redes sociais que visam promover o desenvolvimento da agricultura familiar, através da defesa dos valores implícitos associados à agroecologia, a integração entre pesquisa, extensão e ensino em várias regiões e abrangência no território nacional. Essas redes têm como traço característico principal a adoção de métodos que unem em um só processo a produção de conhecimentos e a sua aplicação prática.

Uma experiência de SAFs importante na construção da agrobiodiversidade local foi realizada até 2007, pela Associação de Produtores Alternativos, doravante APA, localizado no extenso território de Ouro Preto do Oeste (sudoeste da Amazônia) (ABREU; WATANABE, 2008; FIGUEIREDO, 2007; QUOOS, R. D, 2010).

Deve-se salientar neste sentido que a Amazônia é conhecida mundialmente por sua importância ambiental para o equilíbrio do clima, pela abundância e riqueza em termos de biodiversidade, a sua sustentabilidade é considerada uma das soluções para a crise ambiental desencadeada pelo aquecimento global. Entretanto, relativamente pouco se discute à luz de análises de situações concretas, os usos que a população que habita a região faz dos recursos naturais ou o papel desta população diante da conservação ambiental, da agrobiodiversidade e da segurança alimentar (ABREU et al, 2015; ABREU; WATANABE,2008).

Em Ouro Preto do Oeste, a Associação de Produtores Alternativos (APA) desempenhou papel importante no desenvolvimento sustentável e na segurança alimentar local. Após o encerramento das atividades da APA (KOHLER et al, 2011) essa contribuição agora desempenhada por iniciativa dos agricultores familiares teve continuidade, através da adesão às novas organizações sociais. Observou-se que os produtores familiares estabelecem relações com o meio natural com base em conhecimento local tradicional e em novos conhecimentos científicos ecológicos, portanto, suas práticas não são predatórias, mas orientadas para a sustentabilidade agroecológica. Partiu-se do pressuposto que os sistemas agroflorestais quando fortemente diversificados e planejados a partir de uma lógica ecológica são ricos em conhecimento local inovador, se traduz em inovação agroecológica local (FIGUEIREDO, 2007; ABREU et al, 2015).

Buscou-se investigar se os sistemas agroflorestais predominantes adotados pelos produtores familiares, em questão, se constituem em inovação social e agronômica de base ecológica, isto pode ser confirmado pela complexidade em termos de diversidade do sistema. Assim, em síntese neste estudo buscamos qualificar e quantificar a agrobiodiversidade das espécies vegetais e animais.

Assim, objetivou-se com este trabalho, avaliar as potencialidades da diversificação agroecológica das atividades desses agricultores através do confronto entre o que cultivavam e criavam com o que poderiam cultivar e criar conforme as espécies existentes, conhecidas e as espécies pesquisadas, e analisando os desafios para a produção dos produtos oriundos dessas atividades e se a inovação e a diversificação propostas foram adotadas, bem como enumerar as estratégias mais favoráveis a serem adotadas pelos agricultores.

\section{MATERIAL E MÉTODOS}

Ouro Preto do Oeste (Latitude $10^{\circ} 44^{\prime}$ 53" Sul, longitude $62^{\circ} 12^{\prime} 57^{\prime \prime}$ Oeste; a altitude é de $280 \mathrm{~m}$ ), Rondônia, se localiza na região Central do Estado, a $380 \mathrm{~km}$ de Porto Velho, a capital. O clima da região de acordo com a classificação de Koppen-Geiger é do tipo Aw (Peel et al., 2007), com temperatura média de $24,3^{\circ} \mathrm{C}$ e precipitação anual de 1922. Em dezembro de 2007 foi selecionada uma amostra de 29 agricultores familiares de base ecológica inicialmente pertencentes à APA (Associação dos Produtores Alternativos) então com 250 membros.

Entre o final de 2007 e 2016 foram conduzidas pesquisas sobre a produção agroflorestal de produtores ecológicos de Ouro Preto do Oeste, Rondônia, sul da Amazônia. Inicialmente foram entrevistados 29 agricultores de base ecológica; depois, com visitas e contatos com produtores e agentes locais, acompanhou-se a experiência e atualizaram-se os dados da pesquisa. Dos dados coletados selecionamos as atividades por eles conduzidas em sistemas agroflorestais, predominante e cujo padrão local incluí uma diversidade de produções de composição vegetal e animal de pequeno e grande porte, entre as quais: olerícolas, frutíferas, plantas medicinais, piscicultura e apicultura/meliponicultura.

Em cada atividade levantou-se o número de espécies cultivadas ou de criação animal. Simultaneamente, buscou-se na literatura o número de espécies catalogadas em cada atividade e o número de espécies já pesquisadas para as condições de Rondônia e cujo cultivo ou e, criação tivessem sido recomendados. A cada um dos agricultores foi aplicado um questionário semiestruturado contendo questões da seguinte natureza: 1. Atividades agropecuárias praticadas na atualidade; 2. Problemas associados diretamente à produção; 3. Evolução dos sistemas de produção (desde quando se instalou na Unidade de Produção até a data das entrevistas); 4. Vida familiar no momento de instalação em Rondônia e atual; 5. Planos para o futuro da Unidade de Produção;

As respostas dos agricultores foram gravadas, transcritas, tabuladas e analisadas, a partir da metodologia centrada sob a perspectiva cruzada da sociologia compreensiva e da agroecologia (ABREU; BELLON, 2013), adotou-se um conjunto de técnicas de natureza complementar, tais como: observação participante (registro de notas de campo, participação em reuniões); e aplicação de roteiros semiestruturados em amostra de agricultores, técnico 
agroflorestal e agente de desenvolvimento (técnicos da extensão rural).

Dos dados coletados, foram selecionadas as atividades conduzidas em sistemas agroflorestais (SAFs), que inclui a diversidade mencionada anteriormente, levantando-se dentro de cada uma o número de espécies cultivadas e/ou criação. Foi feito um confronto entre as espécies cultivadas ou e, criadas com as espécies existentes e as pesquisadas, para avaliar o potencial para a inovação e diversificação dos sistemas de produção. Analisou-se os desafios para a produção dos produtos oriundos dos sistemas de diversificação, bem como identificou se as estratégias definidas pelos agricultores.

\section{RESULTADOS E DISCUSSÃO}

A APA (Associação dos Produtores Alternativos) foi fundada em 1992 com 29 sócios. Em 2007 contava com 250 associados (FIGUEIREDO, 2007). Os associados da APA compostos por agricultores familiares estabelecidos na região da Grande Ouro Preto do Oeste que compreendem os municípios de Ouro Preto do Oeste, Nova União, Mirante da Serra, Urupá e Vale do Paraíso.

A APA possuí instalações para produção de 2000 potes de palmitos/dia (Sarmento, 2008) e seus produtos são comercializados em municípios de Rondônia, Mato Grosso, Amazonas, São Paulo e Rio de Janeiro (WATANABE; ABREU, 2010). Em 2002 a APA negociava com Alter-Eco uma organização francesa de comércio justo, a entrega de 1500 caixas de palmitos que seriam exportados para a Europa (SARMENTO, 2008) e, assim também outros produtos que beneficiados eram exportados, mel, açaí, etc. A APA mantinha um viveiro com capacidade para produção de 400 mil mudas de pupunha/ano (SARMENTO, 2008) e $500 \mathrm{mil}$ mudas/ano de essências florestais (QUOOS, 2010). Em 2007, a APA teve suas atividades encerradas, com a diretoria sendo processada na justiça por irregularidades financeiro/administrativas (KOHLER et al., 2011).

Em 2010, foi examinada a falência da APA de Ouro Preto do Oeste, buscou se identificar elementos explicativos nas relações entre atores locais. Foi evidenciado a importância da dimensão cultural e econômica da experiência dos produtores familiares. Nesta avaliação destacou se a história social e a cultura política dos produtores familiares e o tipo de capital social construído entorno dos antigos assentamentos da região. Por outro lado, apresentou-se a lógica de funcionamento das instituições envolvidas no desenvolvimento do projeto de consolidação da organização que privilegiam essencialmente a dimensão econômica. As relações entre os diversos atores institucionais são apresentadas como uma rede multiconectada, unindo os níveis local, regional, nacional e internacional. A análise mostra o descompasso entre a visão econômica imediatista das instituições de apoio, voltadas para a produção, e as aspirações dos produtores familiares da APA (KOHLER et al, 2011).

Associa-se a falência a dois fatores interligados: a desconfiança dos produtores da APA em relação aos órgãos de apoio à agricultura familiar (Comissão Executiva do Plano da Lavoura Cacaueira, Agência de Defesa Sanitária Agrosilvopastoril do Estado de Rondônia, Empresa de Assistência Técnica e Extensão Rural) e a ausência dos governos estadual e municipal no grupo de atores do sistema de redes multiconectadas. A crise e a falência da APA não inválida o sucesso da experiência agroecológica e social dos SAFs conduzidos pelos agricultores familiares e essa experiência representa uma resposta social à crise ecológica global (ABREU et al., 2015).

Após o fechamento da APA, os agricultores familiares buscaram a CONAB (Companhia Nacional de Abastecimento) para comercialização de frutas e hortaliças para atender o PAA (Programa de Aquisição de Alimentos). Para a produção de hortaliças, carne de galinha e ovos para venda à $\mathrm{CONAB}$, os agricultores da APA adotaram um sistema de produção denominada PAIS (Produção Agroecológica Integrada e Sustentável) (QUOOS, 2010).

Os SAFs (Sistemas Agroflorestais) conduzidos pelos agricultores familiares de Ouro Preto do Oeste contribuem para a construção da agrobiodiversidade das unidades de produção, redução das emissões de gases de efeito estufa, conservação ambiental e para o fortalecimento da identidade ecológica, conciliadas com o desenvolvimento da agricultura familiar. A evolução detalhada dos sistemas de produção desses agricultores familiares desde a sua chegada em Ouro Preto do Oeste, vindos principalmente de Minas Gerais e Espírito Santo no início da década de 1970 está descrita em Watanabe e Abreu (2010).

Atualmente mais de $80 \%$ dos agricultores agroecológicos mantem como principal atividade econômica a produção leiteira. A produção leiteira se beneficia da agrobiodiversidade oferecida pelos SAFs e, essa atividade está estreitamente integrada aos SAFs, pois o gado leiteiro é criado em sistemas silvopastoris. Além disso, todas as unidades de produção conduzem quintais agroflorestatis com pomares, hortas diversificadas, cultivo de plantas medicinais e criação diversificada de aves e suínos, além de culturas anuais que garantem a segurança alimentar e o bem estar das famílias.

A produção de culturas anuais, no entanto não é suficiente para abastecer as necessidades das famílias, pois dois terços delas precisam comprar de fora da propriedade arroz, feijão e milho. Ouro Preto do Oeste possui atualmente a maior bacia leiteira de Rondônia, apresenta mais de 14 laticínios estabelecidos na região (QUOOS, 2010). Para assessorar os agricultores existem atualmente múltiplas entidades públicas e privadas que proporcionam diversos serviços de apoio tais como cursos de treinamento e capacitação e de assistência técnica e encontros e seminários envolvendo agricultores, extensionistas e outros atores para disseminação e compartilhamento de experiências locais. Ainda assim como será discutido a seguir, de modo geral os técnicos carecem de conhecimentos no campo da agroecologia.

As experiências agroecológicas com SAF's diversificados, criação animal diversificada, quintais agroflorestais estão sendo multiplicadas em centenas comunidades da Amazônia. Essa multiplicação está consolidando esses sistemas de produção economicamente viáveis, preservadoras da biodiversidade e dos recursos naturais, que está conseguindo conciliar o desenvolvimento da agricultura familiar com eliminação do desmatamento e das queimadas e uso de práticas agroecológicas com exclusão do uso de agrotóxicos, como modelos que contribuem para a minimização da crise ecológica global, notadamente a redução das emissões de gases de efeito estufa. 
A tabela 1 indica as condições para a inovação e diversificação dos sistemas de produção dos agricultores familiares, tendo em vista: os números de espécies cultivadas/criadas (a), o número de espécies nativas da Amazônia (b) as espécies nativas de outras regiões brasileiras (c), e o número de espécies pesquisadas para as condições de Rondônia (d). Em 2007, os agricultores familiares cultivavam 22 espécies de hortaliças, todas nativas da Região Centro-Sul do Brasil. Segundo Cardoso (1997) existem 18 espécies de hortaliças nativas da Amazônia; segundo Filgueira (2003) existem 60 espécies de hortaliças convencionais. Em 2007 eram cultivadas 27 espécies de fruteiras em Ouro Preto do Oeste. Silva (2011) revela a existência de 92 espécies de fruteiras nativas da Amazônia. Neste ano, os agricultores familiares cultivavam 19 espécies de plantas medicinais, todas nativas do Centro-Sul. No entanto, Di Stasi e HirumaLima (2002) listam 109 espécies nativas da Amazônia e Lorenzi e Matos (2008) listam 246 espécies nativas da Região Centro-Sul e do Nordeste. Watanabe e Abreu (2010) encontraram 6 tipos de SAFs com culturas principais de cacau, café, seringueira, cupuaçu, açaí e pupunha conduzidos pelos agricultores da APA em Ouro Preto do Oeste.

O palmito pupunha é uma planta nativa da Amazônia e tem grande potencial para substituir o palmito da região Centro Sul, que se encontra atualmente em vias de extinção. A SUFRAMA (2003) já estudou a potencialidades e a viabilidade econômica do cultivo comercial da pupunheira na Amazônia. Ribeiro e Locatelli (2009) pesquisaram 35 espécies para sistemas agroflorestais em condições de Rondônia. Se considerarmos as 92 espécies de frutas nativas da Amazônia, acrescidas de seringueira, cacau, castanha-dopará e pimenta-do-reino, podemos formar 96 SAFs com culturas principais de árvores nativas. Se considerarmos as 77 fruteiras nativas do restante do território nacional, podemos formar 78 SAFs se acrescentarmos o cafeeiro como cultura principal. Todas essas plantas podem ser adaptadas para cultivo agroecológico nas condições de Rondônia, pois muitas delas já foram pesquisadas e aprovadas para cultivo no Estado, tanto nos aspectos técnicos como econômicos.

Tabela 1. Comparação entre espécies cultivadas/criadas com espécies existentes (nativas e de outras regiões) e as pesquisadas para as condições de Rondônia.

\begin{tabular}{lcccc}
\hline \multirow{2}{*}{ Atividades } & & \multicolumn{2}{c}{ Espécies disponíveis } & \\
\cline { 3 - 4 } & $\begin{array}{c}\text { Espécies cultivadas } \\
\text { Criadas (a) }\end{array}$ & Nativas (b) & Outras regiões (c) & $\begin{array}{c}\text { Espécies pesquisadas } \\
\text { (d) }\end{array}$ \\
\hline Olericultura & 22 & 18 & 60 & 45 \\
Plantas medicinais & 19 & 109 & 246 & 41 \\
SAF - fruteiras & 6 & $96 *$ & $78^{* *}$ & 8 \\
SAF- madeireiras & 18 & 250 & 794 & 35 \\
Piscicultura & 3 & 4 & 3 & 9 \\
Apicultura/meliponicultura & 13 & $>300$ & 1 & 1 \\
\hline
\end{tabular}

$* 92+$ seringueira + cacau + castanha-do-pará + pimenta-do-reino $=96$

$* * 77+$ café $=78$

Os agricultores familiares cultivavam 18 espécies de árvores madeireiras/sombreadoras em 2007 (WATANABE; ABREU, 2010). Lorenzi (1998; 2000; 2009) descreve 250 espécies de árvores nativas da Amazônia e 794 espécies de outras regiões brasileiras, todas produtoras de madeira de qualidade, para uso em construção civil, fabricação de móveis, ferramentas, artigos de carpintaria, construção de barcos e remos. A viabilidade econômica dos SAFs madeireiros foi pesquisada por Bentes-Gama (2003) e BentesGama et al. (2005). É possível compor SAFs madeireiros com as 250 espécies nativas da Amazônia e também com as 794 espécies de outras regiões do Brasil. Em 2007 os agricultores familiares da APA criavam 3 espécies de peixes (tilápia, pacu e tambaqui) (WATANABE; ABREU, 2010). Existem 4 espécies de peixes da Amazônia e 3 da Região Centro-Sul. O Governo do Estado de Rondônia (RONDÔNIA, 2007) através do NEAPL (Núcleo Estadual de Arranjo Produtivo Local) criado para atender à piscicultura em 2007, localizado em Pimenta Bueno (188 km de Ouro Preto do Oeste) testou a viabilidade técnica e econômica da criação de 9 espécies de peixes para a Amazônia, incluindo Rondônia. A viabilidade técnica e econômica da atividade já tinha sido avaliada também pela SUFRAMA em 2003. Atualmente são criados tambaqui, tilápia, tambacu, pintado e jatuarana, tendo havido assim diversificação da piscicultura. Quanto à apicultura/meliponicultura os agricultores familiares da APA criavam em 2007, 13 espécies de abelhas (WATANABE; ABREU, 2010). No entanto, existem mais de 300 espécies de abelhas nativas (SILVA; PAZ, 2012) e 1 espécie de abelha europeia africanizada que poderiam ser aproveitadas para a diversificação da apicultura. As potencialidades da meliponicultura foram examinadas recentemente pelo SEBRAE (2015). No entanto após o fechamento da APA, tendo perdido o apoio dessa associação, que era o principal canal de escoamento da produção, atualmente poucos agricultores familiares continuam a produzir mel de abelhas.

Comparando-se esses números, há um vasto espaço para o crescimento, inovação e diversificação dos sistemas de produção desses agricultores, para todas essas atividades, plena oportunidade de se promover integração das propriedades com o ecossistema amazônico. Há um imenso espaço para a pesquisa para introdução do cultivo das 250 espécies de árvores nativas em SAFs e essa pesquisa poderia ser conduzida igualmente por instituições como a EMBRAPA RONDÔNIA CPAF-RO, EMATER-RO, NEAPL e outras. O mesmo pode ser afirmado com relação às 109 espécies de plantas medicinais nativas da Amazônia,18 espécies de hortaliças nativas da Amazônia e as 92 espécies de frutas nativas da Amazônia.

$\mathrm{Na}$ tabela 2 verifica-se as entidades fornecedoras de apoio e assistência técnica para as atividades de olericultura, fruticultura, plantas medicinais, piscicultura e apicultura/meliponicultura em SAFs disponíveis atualmente para os agricultores familiares de Ouro Preto do Oeste. 
Tabela 2. Apoio tecnológico para a produção oriunda de cada atividade disponíveis para agricultores familiares em Ouro Preto do Oeste, Rondônia

\begin{tabular}{|c|c|c|c|c|c|c|c|c|}
\hline Atividade & NEAPL & $\begin{array}{c}\text { SUFRAMA } \\
\text { MAPA }\end{array}$ & ONGs & EMBRAPA & $\begin{array}{c}\text { EMATER- } \\
\text { RO }\end{array}$ & CEPLAC & $\begin{array}{l}\text { SEBRAE- } \\
\text { RO }\end{array}$ & $\begin{array}{l}\text { Associações } \\
\text { Cooperativas }\end{array}$ \\
\hline Olericultura & & & $\mathrm{X}$ & $\mathrm{X}$ & $\mathrm{X}$ & & $\mathrm{X}$ & $\mathrm{X}$ \\
\hline Fruticultura & $X$ & $X$ & $\mathrm{X}$ & $\mathrm{X}$ & $\mathrm{X}$ & $X$ & $\mathrm{X}$ & $X$ \\
\hline Plantas medicinais & & & $\mathrm{X}$ & $\mathrm{X}$ & $\mathrm{X}$ & & $\mathrm{X}$ & $X$ \\
\hline SAFs & & $\mathrm{X}$ & $\mathrm{X}$ & $\mathrm{X}$ & $\mathrm{X}$ & $\mathrm{X}$ & $\mathrm{X}$ & $\mathrm{X}$ \\
\hline Piscicultura & $\mathrm{X}$ & $\mathrm{X}$ & & & $\mathrm{X}$ & & $\mathrm{X}$ & $X$ \\
\hline Apicult/meliponic. & $X$ & & & & $\mathrm{X}$ & & $\mathrm{X}$ & $X$ \\
\hline
\end{tabular}

NEAPL - Núcleo Estadual de Arranjos Produtivos Locais

MAPA - Ministério da Agricultura, Pecuária e Abastecimento

EMBRAPA - Empresa Brasileira de Pesquisa Agropecuária

CEPLAC - Comissão Executiva do Plano da Lavoura Cacaueira

\author{
SEBRAE - Serviço Brasileiro de Apoio às Micro e Pequenas Empresa \\ SUFRAMA - Superintendência da Zona Franca de Manaus \\ ONG - Organização não governamental \\ EMATER - Empresa de Assistência Técnica e Extensão Rural
}

Para os agricultores familiares conseguirem conduzir suas atividades e comercializar a produção uma vez implantadas a inovação baseada na diversificação levantadas no presente trabalho, dependem dos serviços propiciados pelas entidades descritas na Tabela 3. Os agricultores necessitam de assessoria técnica para a implantação da inovação agroecológica, para enfrentar os desafios e para a definição de estratégias favoráveis para a intensificação da diversificação ecológica inovadora. O problema limitante é que nem todos os agricultores familiares têm acesso a esses serviços, muitos dos técnicos desconhecem as práticas e sistemas recomendados para estilos de produção sustentável, portanto é necessário expandir ainda mais a qualificação em treinamento e capacitação de parte importante dos agentes de desenvolvimento local, principalmente no âmbito da agroecologia.

Tabela 3. Serviços prestados aos agricultores pelas entidades públicas e privadas para a produção dos produtos praticada pelos agricultores familiares em Ouro Preto do Oeste, Rondônia

\begin{tabular}{|c|c|c|c|c|c|c|c|c|c|}
\hline Serviços & $\begin{array}{l}\text { EMATER } \\
\text { RO }\end{array}$ & SEBRAE & NEAPL & $\begin{array}{l}\text { ASSOC. } \\
\text { COOP. }\end{array}$ & ONGs & SUFRAMA & MAPA & CEPLAC & MINISTÉRIOS* \\
\hline $\begin{array}{lr}\begin{array}{l}\text { Provimento } \\
\text { regulamentação }\end{array} & \text { de } \\
\text { atividades } & \end{array}$ & & & $\mathrm{X}$ & & & $\mathrm{X}$ & $\mathrm{X}$ & & \\
\hline $\begin{array}{l}\text { Avaliação da } \\
\text { viabilidade } \\
\text { técnica/econômica das } \\
\text { atividades }\end{array}$ & & $X$ & $\mathrm{X}$ & & & $\mathrm{X}$ & $X$ & $X$ & \\
\hline $\begin{array}{l}\text { Estimação do custo de } \\
\text { implantação } \\
\text { previsão de renda das } \\
\text { atividades }\end{array}$ & & $X$ & $X$ & & & $\mathrm{X}$ & $X$ & $X$ & \\
\hline $\begin{array}{l}\text { Provimento de } \\
\text { cursos/treinamentos/ca } \\
\text { pacitação }\end{array}$ & $\mathrm{X}$ & $\mathrm{X}$ & $\mathrm{X}$ & $\mathrm{X}$ & $\mathrm{X}$ & & & $\mathrm{X}$ & \\
\hline $\begin{array}{l}\text { Promoção de apoio e } \\
\text { assistência técnica }\end{array}$ & $\mathrm{X}$ & $X$ & $\mathrm{X}$ & $\mathrm{X}$ & $\mathrm{X}$ & $\mathrm{X}$ & & $X$ & \\
\hline $\begin{array}{l}\text { Promoção de } \\
\text { seminários/dias-de- } \\
\text { campo, encontros com } \\
\text { agricultores, } \\
\text { extensionistas, } \\
\text { pesquisadores } \\
\text { estudantes }\end{array}$ & $\mathrm{X}$ & $\mathrm{X}$ & $\mathrm{X}$ & $\mathrm{X}$ & $\mathrm{X}$ & & & $\mathrm{X}$ & \\
\hline $\begin{array}{l}\text { Apoio e promoção da } \\
\text { agricultura familiar } \\
\text { através de políticas } \\
\text { públicas }\end{array}$ & & & & & & & & & $X$ \\
\hline $\begin{array}{l}\text { MAPA - Ministério da Agri } \\
\text { Social; MMA - Ministério do } \\
\text { Políticas públicas implantada } \\
\text { desenvolvimento local sustent }\end{array}$ & $\begin{array}{l}\text { ra, Pecuá } \\
\text { o Ambier } \\
\text { los Minis }\end{array}$ & $\begin{array}{l}\text { Abastec } \\
\text { DIC }-1 \\
:- \text { Apoi }\end{array}$ & $\begin{array}{l}; \mathrm{ML} \\
\text { rio do } \\
\text { moçã }\end{array}$ & $\begin{array}{l}\text { Ministéri } \\
\text { volvime } \\
\text { gricultu }\end{array}$ & $\begin{array}{l}\text { esen } \\
\text { dústr } \\
\text { iliar; }\end{array}$ & $\begin{array}{l}\text { imento Agrá } \\
\text { Comércio; M } \\
\text { tenção de pr }\end{array}$ & $\begin{array}{l}\text { MDS } \\
\text { - Min } \\
\text { s justo }\end{array}$ & $\begin{array}{l}\text { inistéri } \\
\text { o do } \mathrm{Tr} \\
\text { a os pr }\end{array}$ & $\begin{array}{l}\text { Desenvolvimento } \\
\text { o Emprego } \\
\text { s; -Promoção do }\end{array}$ \\
\hline
\end{tabular}


Os agricultores familiares encontraram também várias dificuldades: Recordam da severa estiagem de 1997, que causou a morte dos cafeeiros levando à quebra de safra e desencadeamento da crise do café, ocasião em que vários agricultores ainda recentemente não haviam conseguido se recuperar. Não tendo colheita da principal atividade (café) para vender, não dispunham de dinheiro para aquisição de novas mudas de cafeeiros. $\mathrm{O}$ ataque repentino de macacos aos frutos dos cupuaçuzeiros reduzindo a colheita; essa atração dos macacos aos frutos do cupuaçuzeiro é confirmado por Lorenzi e Matos (2008).

$\mathrm{O}$ ataque de "vassoura-de-bruxa" aos cacaueiros. O ataque repentino de saúvas desfolhadoras às mudas de árvores para a montagem dos SAFs, obrigando os agricultores a realizarem o replantio das essências florestais. As condições eram mais precárias nos primeiros anos de Rondônia, quando ainda não existiam nenhuma entidade fornecedora de apoio e assistência técnica em agricultura alternativa, bem como de cursos de treinamento e capacitação: Os agricultores tiveram que conduzir suas atividades na base de tentativas e erros, cultivando e criando animais que já conheciam em seus estados de origem, e então diversificar para as culturas e criações amazônicas.

Assim, transferiram para Rondônia o sistema "cabruca" de cultivo do cacaueiro (SAMBUICHI, 2002) mas tiveram que inovar para o cultivo sombreado do cafeeiro (MANCUSO et al., 2013). O escritório da EMATER -RO de Ouro Preto do Oeste foi estabelecido em 1971; a Estação Experimental da EMBRAPA RONDÔNIA CPAF - RO em Ouro Preto do Oeste foi instalada em 1976; o escritório da SEBRAE (Serviço de Apoio às Micro e Pequenas Empresas) mais próximo fica localizado em Ji-Paraná $(42 \mathrm{~km}$ de distância de Ouro Preto do Oeste) (WATANABE; ABREU, 2010) Segundo os pesquisadores Angeletti e Fonseca em 1989, 85\% das hortaliças consumidas em Rondônia eram importadas da Região Centro-Sul. Em 2007, a produção de hortaliças pelos agricultores familiares em Ouro Preto do Oeste se destinava exclusivamente ao autoconsumo. Atualmente alguns agricultores de Ouro Preto do Oeste se especializaram na produção de hortaliças de folhas, sendo que parte deles ampliaram a escala, para venda em feiras-livres, a supermercados, sacolões e exportação para Rio Branco (Acre) e Manaus (Amazonas), além de continuar atendendo o autoconsumo (COSTA et al., 2009).

Para Ribeiro, (2006) e Souza et al., (2011), as frutas nativas, cultivadas ao pleno sol como em SAFs tem um enorme potencial de mercado, pois são apreciadas pela população local, bem como pela população do resto do Brasil e, também no Exterior (NOGUEIRA; SANTANA, 2009). Para dar apoio aos produtores, o governo Estadual estabeleceu em 2007, em Porto Velho um NEAPL (Núcleo
Estadual de Arranjo Produtivo Local). Os problemas que limitam a atividade entre outros são apontados a falta de integração entre o setor produtivo, a agroindústria e os canais de distribuição dos produtos (RONDỐNIA; NEAPL, 2007). A APA foi um importante canal de produção e distribuição de frutas processadas sob a forma de sucos, polpas, geleias, doces, licores (FIGUEIREDO, 2007; WATANABE; ABREU, 2010).

Segundo Kohler et al, 2011, atualmente embora tivessem ficados desamparados com o fechamento da APA há alguns agricultores familiares que tomaram a iniciativa de efetuar o processamento de frutas na própria propriedade, mas desde que dispusessem de recursos financeiros para comprar equipamentos como despolpadoras, conforme os dados atualizados obtidos em 2016. Salienta se que para conduzir SAFs com culturas amazônicas nativas como a seringueira (MARQUES, 2000), açaí (SUFRAMA, 2000; 2003), pupunha (SUFRAMA, 2003), exigiu se dos agricultores toda uma inovação e domínio de uma série de novos conhecimentos tecnológicos, o que foi um grande desafio que tiveram que vencer. Em 2007, de acordo com os dados de Watanabe e Abreu (2010) 31\% dos agricultores familiares da APA pretendiam iniciar a piscicultura. Atualmente trata-se de uma decisão tomada num cenário promissor, pois a atividade além de contar com o apoio de várias entidades, o mercado está bem estruturado e organizado. O peixe e seus derivados sendo produtos altamente perecíveis é essencial que o setor 'produtivo esteja estreitamente integrado com os setores de comercialização, distribuição, transporte e armazenamento, como a pronta disponibilidade de caminhões refrigerados e frigoríficos, de preferência nas proximidades das propriedades dos agricultores. Rondônia tem como vizinhos os Estados de Mato Grosso e Amazonas, para cujos mercados o peixe produzido poderia ser colocado (RONDÔNIA; NEAPL, 2007).

As potencialidades para o aumento da biodiversidade das abelhas nativas a serem criadas em Ouro Preto do Oeste são bastante altas, tendo em vista do número de espécies de abelhas nativas existentes, mais de 300 segundo Silva e Paz (2012). O mel de abelhas nativas produzido com flores das plantas da floresta Amazônica é muito apreciado tanto dentro do território nacional (COSTA et al., 2012; SOUZA et al., 2004; SEBRAE, 2015) como no Exterior. As condições, desafios e estratégias a serem adotadas para a produção de hortaliças, frutas, produtos agroflorestais (SAFs), plantas medicinais, pescados e mel de abelhas uma vez implantadas a inovação e a diversificação são viabilizadas pela disponibilidade dos recursos da biodiversidade e de políticas públicas e assistência técnica levantados no presente trabalho e sumarizadas são observadas na Tabela 4 e no Quadro 1.

Tabela 4. Condições, desafios e estratégias para a produção agropecuária de agricultores familiares de Ouro Preto do Oeste, Rondônia

\begin{tabular}{lcccc}
\hline Atividade & Condições & Desafios & Estratégias & Provedores de apoio \\
\hline Olericultura & $1,2,3,4,5,8$ & $1,2,5,6,13$ & $1,2,3,4,5,6,7$ & $1,2,3,6,7$ \\
Fruticultura & $5,6,7,8,9$ & $3,4,5,6$ & $1,7,8,9$ & $1,2,3,4,5,6,7$ \\
SAFs & $10,11,12$ & 7,8 & $1,7,10,11$ & $1,2,3,6,7$ \\
Plantas medicinais & 13 & 9,10 & $1,7,13,14$ & $1,2,3,6,7$ \\
Piscicultura & $10,14,15$ & 7,11 & $1,7,15$ & $1,2,4,5,6,7$ \\
Apicult/melípona. & $2,8,10,16$ & $6,7,12,13$ & $1,5,7,12$ & $1,2,5,6,7$ \\
\hline
\end{tabular}


Quadro 1. Sumarização das condições, desafios e estratégias para a produção agropecuária de agricultores familiares de Ouro Preto do Oeste, Rondônia

\begin{tabular}{|c|c|}
\hline$\underline{\text { Condições }}$ & $\begin{array}{l}\text { 1. Alta temperatura, alta pluviosidade } \\
\text { 2. Alto custo de produção } \\
\text { 3. Falta de sementes de qualidade } \\
\text { 4. Competição com hortaliças importadas } \\
\text { 5. Falta de mão de obra especializada } \\
\text { 6. Sazonalidade da produção } \\
\text { 7. Baixo nível tecnológico do agricultor } \\
\text { 8. Baixa qualidade do produto } \\
\text { 9. Falta de integração entre os setores produtivo, agroindústria e distribuição } \\
\text { 10. Poucas espécies cultivadas/criadas } \\
\text { 11. Crise do café - estiagem de } 1997 \\
\text { 12. Vassoura de bruxa que atacou os cacaueiros } \\
\text { 13. Pequena escala de produção para atender apenas o autoconsumo } \\
\text { 14. Tendência a criar apenas o tambaqui } \\
\text { 15. Excesso de produção de tambaqui } \\
\text { 16. Baixa produtividade }\end{array}$ \\
\hline$\underline{\text { Desafios }}$ & $\begin{array}{l}\text { 1. Produção de hortaliças de alta qualidade, nas condições de Rondônia, a preços competitivos } \\
\text { 2. Falta de treinamento da mão de obra } \\
\text { 3. Ampliação do período de frutificação } \\
\text { 4. Falta de melhoramento genético para obter plantas com frutificação mais precoce ou mais } \\
\text { tardia } \\
\text { 5. Elevação do nível tecnológico do produtor e da mão de obra } \\
\text { 6. Melhoramento da qualidade do produto } \\
\text { 7. Aumento do número de espécies cultivadas/criadas } \\
\text { 8. Superação da crise do café e do ataque de vassoura da bruxa } \\
\text { 9. Produção em escala comercial } \\
\text { 10. Conversão para o sistema orgânico certificado } \\
\text { 11. Diminuição da produção de tambaqui } \\
\text { 12. Aumento da produtividade } \\
\text { 13. Redução do custo de produção }\end{array}$ \\
\hline Estratégias & $\begin{array}{l}\text { 1. Diversificação das espécies de culturas/criações com adoção de tecnologias inovadoras } \\
\text { 2. Cultivo de hortaliças entre os meses de abril e setembro } \\
\text { 3. Escolha de variedades adequadas para as condições de Rondônia para obtenção de hortaliças } \\
\text { de alta qualidade } \\
\text { 4. Produção de sementes na propriedade ou importação de sementes da Região Centro-Sul } \\
\text { 5. Redução dos custos de produção } \\
\text { 6. Treinamento da mão de obra e do agricultor } \\
\text { 7. Obtenção da certificação do sistema de produção pela ACS Amazônia Associação de } \\
\text { 8ertificação Sócio participativa da Amazônia } \\
\text { 8. Manejo para ampliar o período de frutificação } \\
\text { 9. Seleção de variedades mais produtivas que produzam frutas de melhor qualidade, que } \\
\text { frutifiquem mais precocemente ou mais tardiamente } \\
\text { 10. Obtenção de financiamento para compra de novas mudas de cafeeiros } \\
\text { 11. Controle da vassoura de bruxa com manejo } \\
\text { 12. Seleção de colmeias mais produtivas e que produzam mel de melhor qualidade } \\
\text { 13. Treinamento para aprendizagem da tecnologia de produção, beneficiamento e controle de } \\
\text { qualidade } \\
\text { 14. Implantação do sistema orgânico de produção certificada } \\
\text { 15. Evitar criação exclusiva de tambaqui }\end{array}$ \\
\hline$\underline{\text { Provedores de apoio }}$ & $\begin{array}{ll}\text { 1. } & \text { EMATER - RO - Empresa de Assistência Técnica e Extensão Rural } \\
\text { 2. } & \text { SEBRAE - RO - Serviço Brasileiro de Apoio às Micro e Pequenas Empresas } \\
\text { 3. } & \text { EMBRAPA - RO - Empresa Brasileira de Pesquisa Agropecuária } \\
\text { 4. } & \text { SUFRAMA - Superintendência da Zona Franca de Manaus } \\
\text { 5. } & \text { NEAPL - Núcleo Estadual de Arranjos Produtivos Locais } \\
\text { 6. } & \text { ONGs - Organização não Governamental } \\
\text { 7. } & \text { ASSOCIAÇÕES E COOPERATIVAS }\end{array}$ \\
\hline
\end{tabular}


Assim, a experiência social com sistemas alimentares agroflorestal mostra que esses produtores se encontram inseridos em redes territoriais de inovação local que articulam famílias produtoras em diferentes estágios de transição, tratase de dispositivos coletivos adequados para a criação de ambientes voltados para o aprendizado mútuo e disseminação de formas harmônicas de convivência com a natureza, mas as instituições necessitam ampliar o número de profissionais com conhecimento em agroecologia. As experiências estudadas são resultado de uma trajetória social que responde positivamente aos impactos sociais, econômicos e ecológicos da produção convencional ainda predominante, que se constitui num fator crítico para a sustentabilidade e, em especial para a agricultura familiar.

\section{CONCLUSÕES}

Existe grande diferença entre o número de espécies cultivadas/criadas e o número de espécies existentes e as pesquisadas para as condições de Rondônia. Assim havendo espaço para a inovação baseada na diversificação agroecológica das atividades de olericultura, fruticultura, SAFs, cultivo de plantas medicinais, piscicultura e apicultura ou meliponicultura.

O desenvolvimento sustentável do território rural da Grande Ouro Preto dependerá em grande parte da consolidação das alternativas de produção agroflorestal de políticas para a gestão ambiental integrada, sendo fundamental ampliar e fortalecera capacidade institucional em aprimorar e consolidar políticas locais de base sustentável para a agricultura familiar.

\section{REFERÊNCIAS}

ABREU, L. S. de; BELLON, S. The dynamics and decomposition of agro ecology in Latin America. In: HALBERG, N; MULLER, A. (Ed.). Organic agriculture for sustainable livelihoods. Routeledge, 2013. p. 223-245.

ABREU, L. S. de; SANTOS, A.; WATANABE, M. A. Agricultores familiares da região sul da Amazônia brasileira: uma contribuição à crise ecológica global. In: CONGRESO LATINOAMERICANO DE AGROECOLOGÍA, 5., 2015, La Plata. La agroecología: un nuevo paradigma para redefinir la investigación, la educación y la extensión para una agricultura sustentable: memorias... La Plata: Sociedad Científica Latinoamericana de Agroecología, 2015. 6 p.

ABREU, L. S. de; WATANABE, M. A. Agroforestry systems and food security among smallholder farmers of the brazilian Amazon: a strategy for environmental global crisis. In: ISOFAR SCIENTIFIC CONFERENCE, 2., 2008, Modena. Cultivating the future based on science. Modena: ISOFAR, 2008. v.2, p.472.

ANGELETTI, M. da P.; FONSECA, A.F.A. da. Instruções técnicas para o cultivo comercial de hortaliças em Rondônia. Porto Velho: EMBRAPA-UEPAE Porto Velho, 1989. 67p. (EMBRAPA-UEPAE Porto Velho. Circular Técnica, 11).
BENTES-GAMA, M. de M. Análise técnica e econômica de SAFs em Machadinho d'Oeste, Rondônia. 2003. 126 p. Tese (Doutorado) -- Universidade Federal de Viçosa, Viçosa, 2003.

BENTES-GAMA, M. de M. et al. Análise econômica de sistemas agroflorestais na Amazônia Ocidental, Machadinho D'Oeste - RO. Revista Árvore, Viçosa, v. 29, n. 3, p. 401-411, 2005.

CARDOSO, M. O. (Coord.). Hortaliças não-convencionais da Amazônia. Brasília, DF: Embrapa-SPI; Manaus: EmbrapaCPAA, 1997. 150 p. il. color.

COSTA, T. V.; FARIAS, C. A. G.; BRANDÃO, C. dos S. Meliponicultura em comunidades tradicionais do Amazonas. Revista Brasileira de Agroecologia, Porto Alegre, v. 7, n. 3, p. $106-115,2012$.

COSTA, D. L.; COELHO NETTO, R. A; CORAL, S.C.T.; REIS, L. M. Agroecologia, autoconsumo e comercialização de produtos da agricultura familiar na Amazônia. In: Reunião Anual da SBPC, 61. 2009, Manaus. Anais... Manaus: SBPC, 2009. 2 p.

DI STASI, L. C.; HIRUMA-LIMA, C. A. Plantas medicinais na Amazônia e na Mata Atlântica. São Paulo: Fundação Editora da Universidade Estadual Júlio de Mesquita Filho, 2002. 592 p.

FILGUEIRA, F. A. R. Novo manual de olericultura: agro tecnologia moderna na produção e comercialização de hortaliças. 2. ed. Viçosa: Editora da UFV, 2003. 412 p.

FIGUEIREDO, L. D. Produzir sem destruir: a experiência da Associação dos Produtores Alternativos de Ouro Preto do Oeste (RO). In: PETERSEN, P.; DIAS, A. (Org.) Construção do conhecimento agroecológico: novos papéis, novas identidades. Recife, Articulação Nacional em Agroecologia, 2007. In: Caderno do II Encontro Nacional de Agroecologia, 2007, Recife, p. 73-87

KOHLER, F.; ISSBERNER, L. R.; LENA, P.; MARCHAND, G. Falência é fracasso? O caso da Associação dos Produtores Alternativos de Ouro Preto do Oeste, Rondônia, Brasil. Boletim do Museu Paraense Emílio Goeldi, Belém, v. 6, n. 2, p. 1-13, 2011.

LORENZI, H. Árvores brasileiras. 3. ed. Nova Odessa: Instituto Plantarum de Estudos da Flora, 2000. v. 1, 2000. $384 \mathrm{p}$.

LORENZI, H. Árvores brasileiras: manual de identificação e cultivo de plantas arbóreas nativas do Brasil. Nova Odessa: Instituto Plantarum de Estudos da Flora, 1998. v. 2, 384 p.

LORENZI, H. Árvores brasileiras: manual de identificação e cultivo de plantas arbóreas nativas do Brasil. Nova Odessa: Instituto Plantarum de Estudos da Flora, 2009. v.3, 384 p.

LORENZI, H.; MATOS, F. J. A. Plantas medicinais no Brasil: nativas e exóticas. Nova Odessa: Instituto Plantarum de Estudos da Flora Ltda., 2008. 544 p. 
MANCUSO, M. A. C.; SORATTO, R. P.; PERDONÁ, M. J. Produção de café sombreado. Produção de café sombreado. Colloquium Agrariae, Presidente Prudente, v. 9, n. 1, p. 3144, 2013

MARQUES, J. R. Seringueira. Transcrito do Jornal CEPLAC Notícias. Brasília, dez. 2000.

NOGUEIRA, A. K. M.; SANTANA, A. C. de Análise de sazonalidade de preço de varejo de açaí, cupuaçu e bacaba no Estado do Pará. Revista de Estudos Sociais, Cuiabá, ano 11, v. 1, n. 21, p. $7-22,2009$.

PEEL, M. C.; FINLAYSON, B. L.; MCMAHON,T.A. Updated world maps of the Köppen-Geiger Climate Classification Hidrol Earth Syst. Sci.11, 1633-1644-0. 2007.

QUOOS, R. D. Transição agroecológica em Rondônia: a Associação dos Produtores Alternativos de Ouro Preto do Oeste. 2010. 152 p. Dissertação (Mestrado) -- Universidade Federal do Rio Grande do Sul, Porto Alegre, 2010.

RIBEIRO, G. D. Fruticultura tropical: uma alternativa para a agricultura de Rondônia. Porto Velho: EMBRAPA Rondônia CPAF-RO, 2006, 15 p. (Documentos, 109).

RIBEIRO, M. S. de M.; LOCATELLI, M. Estudo preliminar de sistemas agroflorestais no Distrito de Triunfo, Candeias do Jamari, Rondônia. In: CONFERÊNCIA DO SUBPROGRAMA DE CIÊNCIA E TECNOLOGIA, SPC\&T, Fase II/PPG7. Anais... Brasília, CNPq. 2009. p. $505-509$.

RONDÔNIA (Estado). Núcleo Estadual de Apoio aos Arranjos Produtivos Locais de Rondônia. Arranjo produtivo local: fruticultura de Porto Velho - Rondônia. Porto Velho: Plano de Desenvolvimento Preliminar, 2007. 36 p.

RONDÔNIA (Estado). Núcleo Estadual de Apoio aos Arranjos Produtivos Locais de Rondônia. Arranjo produtivo local da piscicultura de Pimenta Bueno - Rondônia. Pimenta Bueno: Plano de Desenvolvimento Preliminar, 2007. 5 p.

SAMBUICHI, R. H. R. Fitossociologia e diversidade de espécies arbóreas em cabruca (Mata Atlântica raleada sobre plantação de cacau) na região Sul da Bahia, Brasil. Acta Botânica Brasílica. Porto, Alegre, v. 16, n. 1, p. 89-101, 2002.

SARMENTO, S. S. de O. A representação social de uma trajetória: Associação dos Produtores Alternativos de Ouro Preto do Oeste-RO. 2008. 112 p. Dissertação (Mestrado) -Fundação Universidade Federal de Rondônia, Porto Velho, 2008.

SEBRAE. Potencialidades da meliponicultura: criação de abelhas nativas 2015 Disponível em: < http://www.sebrae.com.br/sites/PortalSebrae/artigos/potencial idades-da-meliponicultura-criacao-de-abelhasnativas,36267683f9cbe410VgnVCM1000003b74010a.RCD\# 0> . Acesso em 26 jan. 2017
SILVA, S. Frutas da Amazônia brasileira. São Paulo, Metalivros, 2011. 280 p.

SILVA. W. P.; PAZ, J. R. C. Abelhas sem ferrão: Muito mais do que uma importância econômica. Natureza online, Santa Teresa, v. 10, n. 3, p. 146-152, 2012.

SOUZA, M. P. de; SILVA, T. N.; PEDROZO, E. Á.; SOUZA FILHO, T. A. O produto florestal não madeirável (PFNM) amazônico açaí nativo: Proposição de uma organização social baseada na lógica de cadeia e rede para potencializar a exploração local. Revista de Administração e Negócios da Amazônia, Porto Velho, v. 3, n. 2, p. 44-57, 2011.

SOUZA, R. C. S.; YUYAMA, L. K. O.; AGUIAR, J. P. L.; OLIVEIRA, F. P. M. Valor nutricional do mel e pólen de abelhas sem ferrão da região amazônica. Acta Amazônica, Manaus v. 34, n. 2, p. 333-336, 2004.

SUPERINTENDÊNCIA DA ZONA FRANCA DE MANA. Projeto potencialidades regionais - Rondônia, estudo da viabilidade econômica - açaí, áreas de concentração da produção de açaí (AM, AC, AP, RO). Manaus: SUFRAMA, 2000. 66 p.

SUPERINTENDÊNCIA DA ZONA FRANCA DE MANAUS. Projeto potencialidades regionais, estudo da viabilidade econômica - açaí, áreas de concentração da produção de açaí (AM, AC, AP, RO). Manaus, SUFRAMA, 2003. $22 \mathrm{p}$.

SUPERINTENDÊNCIA DA ZONA FRANCA DE MANAUS. Projeto potencialidades regionais - Rondônia, estudo da viabilidade econômica - piscicultura. Manaus: SUFRAMA, 2003. v. 8, 72 p.

SUPERINTENDÊNCIA DA ZONA FRANCA DE MANAUS. Projeto potencialidades regionais, estudo da viabilidade econômica - palmito de pupunheira. Manaus: SUFRAMA, 2003. 36 p.

WATANABE, M. A.; ABREU, L. S. de. Estudo agroecológico e social de agricultoras familiares de base ecológica no Sudoeste da Amazônia (Ouro Preto do Oeste, Rondônia). Jaguariúna: Embrapa Meio Ambiente, 2010, 58 p. (Documentos, 81). 